\title{
Shielding of the electromagnetic field of a coplanar waveguide by a metal film: Implications for broadband ferromagnetic resonance measurements
}

Matthieu Bailleul

Citation: Appl. Phys. Lett. 103, 192405 (2013); doi: 10.1063/1.4829367

View online: https://doi.org/10.1063/1.4829367

View Table of Contents: http://aip.scitation.org/toc/apl/103/19

Published by the American Institute of Physics

\section{Articles you may be interested in}

Ferromagnetic resonance linewidth in metallic thin films: Comparison of measurement methods Journal of Applied Physics 99, 093909 (2006); 10.1063/1.2197087

Strong asymmetry of microwave absorption by bilayer conducting ferromagnetic films in the microstrip-line based broadband ferromagnetic resonance Journal of Applied Physics 106, 043903 (2009); 10.1063/1.3187547

Vector network analyzer ferromagnetic resonance of thin films on coplanar waveguides: Comparison of different evaluation methods

Journal of Applied Physics 101, 074505 (2007); 10.1063/1.2716995

Coplanar waveguide based ferromagnetic resonance in ultrathin film magnetic nanostructures: Impact of conducting layers

Journal of Applied Physics 116, 053901 (2014); 10.1063/1.4891734

A coplanar waveguide permeameter for studying high-frequency properties of soft magnetic materials Journal of Applied Physics 96, 2969 (2004); 10.1063/1.1774242

Spin wave contributions to the high-frequency magnetic response of thin films obtained with inductive methods Journal of Applied Physics 95, 5646 (2004); 10.1063/1.1697641

\section{PHYSICS TODAY}

MANAGER'S GUIDE

Accelerate R\&D with

Multiphysics Simulation

\section{READ NOW}

PRESENTED BY

マ๐ $\subset$ MSOL 


\title{
Shielding of the electromagnetic field of a coplanar waveguide by a metal film: Implications for broadband ferromagnetic resonance measurements
}

\author{
Matthieu Bailleul ${ }^{\text {a) }}$ \\ Institut de Physique et Chimie des Matériaux de Strasbourg, UMR 7504 CNRS-Université de Strasbourg, \\ 23 rue du Loess, BP 43, 67034 Strasbourg Cedex 2, France
}

(Received 1 July 2013; accepted 23 October 2013; published online 8 November 2013)

\begin{abstract}
We show that the propagation of microwave fields along a planar transmission line is strongly modified when a conducting film is brought close to it. The effect is attributed to the shielding of the electrical and/or magnetic microwave fields which is shown to occur over a wide range of parameters (microwave frequency, film square resistance, transverse dimensions of the waveguide). This is illustrated by finite-element electromagnetic simulations and interpreted using a distributed impedance model. We discuss the implications of this phenomenon for broadband measurements of ferromagnetic resonance realized by placing a ferromagnetic metal film above a coplanar waveguide. (C) 2013 AIP Publishing LLC. [http://dx.doi.org/10.1063/1.4829367]
\end{abstract}

Most spintronics devices rely on thin ferromagnetic metal films (Fe, Co, Ni, and their alloys). Because these devices are often to be operated in the high frequency $(\mathrm{GHz})$ regime, in which the phenomenon of ferromagnetic resonance (FMR) occurs, it is essential to characterize precisely their microwave magnetic response. For this purpose, broadband techniques using transmission lines have been developed in the last 15 years. In such techniques, a portion of the ferromagnetic film is placed close to the surface of a planar transmission line, in most cases, a coplanar waveguide (CPW). The microwave magnetic field $\mathbf{H}$ delivered by the CPW excites the precession of the magnetization of the film which, in turn, induces an additional microwave voltage on the CPW. In practice, the FMR signal is extracted from the reflection-transmission coefficients of the loaded transmission line, measured as a function of the external magnetic field and the microwave frequency. The technique is referred to as CPW-FMR, ${ }^{1,2}$ network-analyzer FMR, ${ }^{3-5}$ broad-band $\mathrm{FMR}^{6}{ }^{6}$ or pulse-induced microwave magnetometer ${ }^{7}$ in its time-domain implementation. Although the technique is relatively straightforward to implement, the extraction of the magnetic parameters from the measured microwave response requires some precautions. In particular, proper de-embedding techniques should be used to treat the propagation of the microwaves along the $\mathrm{CPW},{ }^{3}$ and the transverse inhomogeneity of the microwave magnetic field should be accounted for in the interpretation of the FMR frequencies and linewidth. ${ }^{8}$ Another very important point was recognized only very recently: The films to be measured are usually strongly conducting [typically $30 \Omega /$ square for a $10 \mathrm{~nm}$ thick permalloy $\left(P y=N i_{80} F e_{20}\right)$ film], which results in a strong modification of the distribution of the electric and magnetic microwave fields close to the film. This was described by Kostylev in terms of a shielding of the microwave field by eddy currents flowing along the conducting film: ${ }^{9}$ calculations in simplified plane wave propagation geometries indicate that this effect occurs for a wide range of film thicknesses, even much below typical microwave skin depth

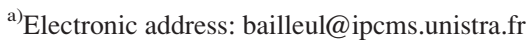

which is of the order of several hundreds of nanometers for the materials usually investigated by CPW-FMR. Moreover, it has been shown that the eddy currents are responsible for the excitation of FMR modes having a non-uniform distribution of the oscillating magnetization across the film thickness ${ }^{6,10}$ (so-called perpendicular standing spin wave modes) which could not be excited in the absence of shielding.

In this Letter, we build upon these previous works by addressing the phenomenon of shielding in a realistic coplanar waveguide geometry with the help of finite element electromagnetic simulations. Then a simple interpretation is given within the conventional formalism of transmission lines. Finally, we discuss how the phenomenon of shielding could be accounted for in broadband FMR measurements.

The geometry used for the simulation is shown in Fig. 1(a). It consists of a conventional coplanar waveguide (a $t=50 \mu \mathrm{m}$ thick $w=350 \mu \mathrm{m}$ wide track surrounded by two lateral ground planes with gaps $g=170 \mu \mathrm{m}$ ) complemented with an additional ground plane and two vertical walls enclosing a $e=200 \mu \mathrm{m}$ thick substrate of relative permittivity $\epsilon_{r}=3.55$. This geometry, known as a channelized CPW (CCPW), has the advantage of allowing single mode operation up to relatively high frequencies, due to the full metallization around the substrate providing high cut-off frequencies for parasitic non-TEM modes. ${ }^{11}$ The design of Fig. 1(a) mimics the transverse cross-section of test boards fabricated using conventional printed circuit board techniques over a RO4003 substrate (Rogers Corp.), which demonstrates single mode operation up to $50 \mathrm{GHz} .{ }^{11}$ The space above the CPW is enclosed in an air box of thickness $h_{\text {air }}$ $=340 \mu \mathrm{m}$. All the metallic boundaries defining the CCPW are assumed to be perfectly conducting. A metal film of square resistance $R_{s q}$ is located above the CPW at a height $h=30 \mu \mathrm{m} .{ }^{17}$ It extends laterally up to the vertical walls of the airbox, which provides an explicit electrical connection to ground. Because the focus is on the conductivity of the metal film, it is assumed to be nonmagnetic $\left(\mu_{r}=1\right)$ and the presence of its substrate is not accounted for (i.e., $\epsilon_{r}$ is set to 1 above the film). 


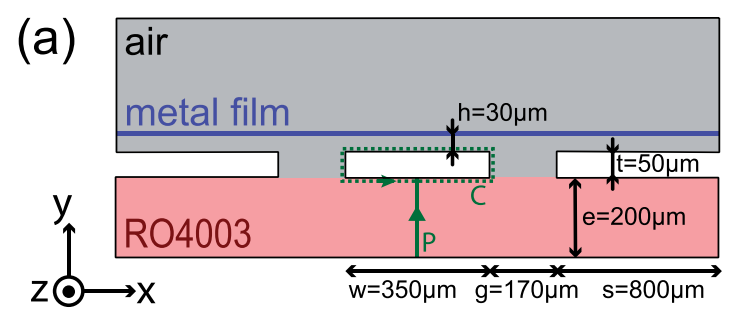

(b) Au1 $\mu$ m, 100nm Py $100 \mathrm{~nm}, 10 \mathrm{~nm}$
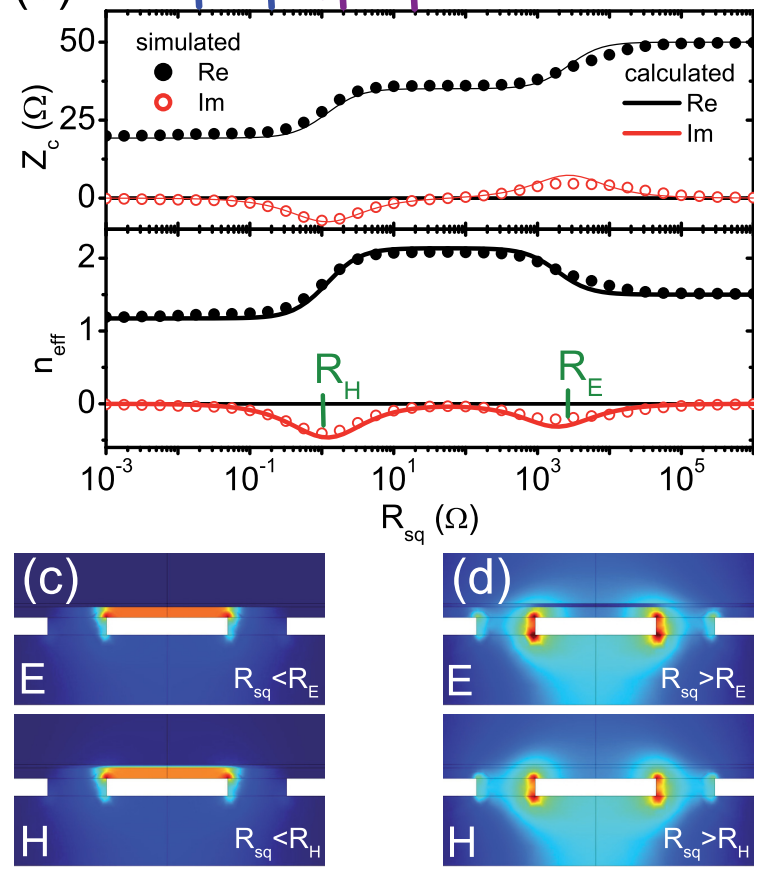

FIG. 1. (a) Geometry of the finite element simulation. (b) Results of the simulation of the propagation at a frequency of $2 \mathrm{GHz}$ (dots). The real and imaginary parts of the characteristic impedance $Z_{c}$ (top) and the effective propagation index $n_{\text {eff }}$ (bottom) are shown as a function of the resistance per square of the film $R_{s q}$. The lines are the results of the model of Fig. 2. (c) Amplitude maps of the microwave electric and magnetic fields for $R_{s q}=0.1 \Omega$. The region around the central track has been zoomed in for clarity. Color scale: blue (zero) to red (maximum amplitude). (d) Same for $R_{s q}=100 \mathrm{k} \Omega$.

The simulations are carried out using COMSOL Multiphysics (version 4.0a, RF module) in the mode 2D/Electromagnetic Wave/Mode Analysis. They consist in solving the Maxwell equations describing the propagation of electromagnetic waves along an infinite transmission line having a cross-section as sketched in Fig. 1(a). The microwave frequency is fixed at $f=2 \mathrm{GHz}$ and the eigenvalue problem obtained after meshing the cross section into about 2000 triangles is solved, thus providing the allowed propagation mode and its propagation wave-vector $k_{z}$. As expected for the chosen design, one obtains only one propagation mode which is predominantly $\mathrm{TEM}\left(E_{z} \simeq 0, H_{z} \simeq 0\right)$. From the simulation, the transverse distributions of the electric $[\mathbf{E}(x, y)]$ and magnetic $[\mathbf{H}(x, y)]$ fields are extracted together with the propagation index $n_{e f f}=c k_{z} / 2 \pi f$ and the characteristic impedance $Z_{c}=V / I$ with $V=\int_{P} E_{y} . d y$ and $I=\oint_{\mathcal{C}} \mathbf{H} . \mathbf{d l}$ [see the integration paths $P$ and $C$ in green in Fig. 1(a)]. Note that $n_{\text {eff }}$ and $Z_{c}$, which would be purely real if only perfect metals were involved, are expected to have non-zero imaginary parts due to the finite conductivity of the metal film. Figure 1(b) shows the real and imaginary parts of $n_{\text {eff }}$ and $Z_{c}$ as a function of the resistance per square of the film $R_{s q}$, varied between $10^{-3}$ and $10^{6} \Omega$. One distinguishes clearly three plateaus for the real parts of $n_{\text {eff }}$ and $Z_{c}$. These plateaus are separated by transition regions in which the real parts of $n_{\text {eff }}$ and $Z_{c}$ vary smoothly while their imaginary parts show peaks. For $R_{s q}>R_{E} \simeq 2.5 \mathrm{k} \Omega$, one recovers the value $Z_{c}^{C P W}=Z_{0}=50 \Omega$ and $n_{e f f}^{C P W}=1.5$ targeted in the design of the CCPW (i.e., no effect of the conductance of the film). On the other hand, for $R_{s q}<R_{H} \simeq 1 \Omega$ (i.e., for a strongly conducting film), $Z_{c}$ and $n_{\text {eff }}$ are reduced to $20 \Omega$ and 1.2, respectively. For intermediate values of film resistance $\left(R_{H}<R_{s q}<R_{E}\right)$, one observes an intermediate value of $Z_{c}$ (35 $\Omega$ ) and a higher value of $n_{\text {eff }}$ (2.1). Interestingly, different distributions of $\mathbf{E}$ and $\mathbf{H}$ are observed for each of the three plateaus [see Figs. 1(c) and 1(d)]. For $R_{s q}>R_{E}$, both $\mathrm{E}$ and $\mathrm{H}$ appear to be concentrated in the gaps between the center track and the lateral ground planes ${ }^{18}$ [Fig. 1(d)], while for $R_{s q}<R_{H}$, they become strongly concentrated in the air gap between the center track and the metal film [Fig. 1(c)]. For $R_{H}<R_{s q}<R_{E}$, the distributions of the electric and magnetic fields are different from each other: $\mathbf{E}$ is concentrated above the central track, exactly as in the low resistance regime, while $\mathbf{H}$ is distributed around the central track, as in the high resistance regime (not shown). These results are interpreted as follows: In the high resistance regime, the effect of the metal film is negligible and one recovers the characteristics of the unperturbed CCPW [see Fig. 2(a)]. In the low resistance limit, the metal film acts as a perfect ground plane which dominates over the other ground planes because of its proximity to the signal track. The propagation mode is then very close to that of a transmission line consisting of two parallel plates with air in between [PP, see Fig. 2(b)]. The propagation parameters of this transmission line are easily evaluated as $n_{e f f}^{P P} \simeq 1$ and $Z_{c}^{P P} \simeq \sqrt{\mu_{0} / \epsilon_{0}} h / w=32 \Omega .^{12} \mathrm{In}$ this regime, the metal film acts as a perfect shield, i.e., it carries currents and charges opposite to those existing in the signal track so that the microwave field above the film is zero. Apparently a film of intermediate resistance allows for the shielding of the electric field but not for that of the magnetic field.

Let us now model the observed behavior within the standard formalism of transmission lines. For a short length $\delta$ of the CCPW loaded with the metal film, the CPW propagation mode is modeled by combining a series inductance (a)

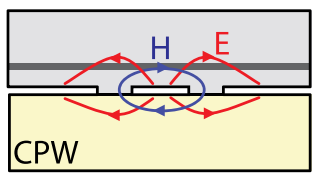

(c)

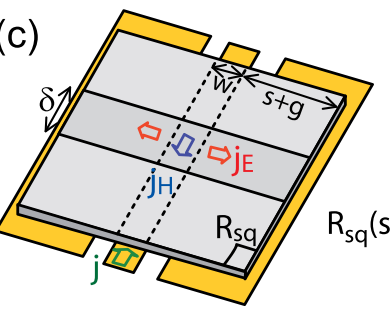

(d)

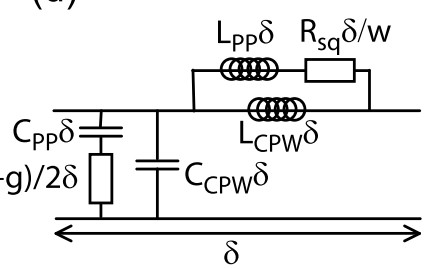

FIG. 2. (a) and (b) Sketches of the CPW and PP modes. (c) Sketch of the current $j_{H}$ (resp. $j_{E}$ ) which has to flow along the metal film to shield the magnetic field (resp. the electric field). (d) Distributed impedance model accounting for the competition between the CPW and PP modes. 
$L_{C P W} \delta$ and a parallel capacitance $C_{C P W} \delta$. To account for the competition with the PP mode, these are put in parallel with the inductance and capacitance of the PP mode [see Fig. 2(d)]. Series resistors are included in these two additional branches to account for the electrical resistance opposed by the metal film to the shielding currents: To confine the magnetic field below the metal film, a current $j_{H}$ extending approximately over the width $w$ should flow along the film in the direction opposite to that of the central track current $j$ [see Fig. 2(c)]. This translates into a resistance $R_{s q} \delta / w$ adding to the inductive parallel-plate branch. In a similar way, the shielding of the electric field requires currents $j_{E}$ returning towards both lateral walls, which translates into a resistance $R_{s q}(s+g) / 2 \delta$ adding to the parallel branch. The lines in Fig. 1(b) show the global characteristic impedance and effective index deduced from the model of Fig. 2(d) ${ }^{19}$ They reproduce extremely well the result of the simulation, which suggests that despite its simplicity the proposed model captures the physics of the observed phenomenon of microwave shielding. Let us briefly comment on the intermediate regime where only the electric field is shielded. Due to the fact that the spatial distributions of the electrical and magnetic field are different from each other, it is possible to tune the capacitance and inductance per unit length independently from each other by changing some dimensions in the structure, which allows one to get very high values of $n_{\text {eff }}$ while keeping $Z_{c}$ at $50 \Omega$. Such structures are known in the microwave community as slow-waves lines, ${ }^{13}$ they are used for reducing the footprint of delay lines and for reducing substrate losses in interconnects on non-insulating substrates (e.g., silicon). Interestingly, the model described here allows one to understand very simply the architectures used to obtain slow-waves: semiconducting layer located below the CPW with a suitable doping (such that its square resistivity falls in the $R_{E}<R_{s q}<R_{H}$ range), ${ }^{14}$ or array of metal strips oriented perpendicular to the CPW axis [such that $j_{E}$ currents are allowed, whereas $j_{H}$ currents are forbidden, see Fig. 2(c) $]^{15}$

For further simplification, it is possible to write in a compact way the maximum film resistance $R_{E}$ (resp. $R_{H}$ ) allowing for the shielding of the electric (resp. magnetic) field. Assuming $C_{P P} \gg C_{C P W}$ and $L_{P P} \ll L_{C P W}$, one obtains $R_{E}=Z_{0} \lambda / \pi(s+g)$ and $R_{H}=Z_{0} w / 2 \pi \lambda$, where $\lambda$ is the CPW propagation wavelength. Thus, the relevant shielding regime can be determined from a simple ratio of dimensions. Fig. 3 shows a phase diagram of the phenomenon of shielding. The transition film resistances $R_{E}$ and $R_{H}$ are plotted as a function of the frequency for three different sets of dimensions ( $w, s$ $+g$ ) representative of the transmission lines used for broadband FMR. The first set, corresponds to the design investigated in this paper, typical of ultra-broadband transmission lines on printed circuit boards. The second set corresponds to a relatively narrow coplanar waveguide on a silicon or alumina substrate, typical of CPWs fabricated using thin film lithography techniques. ${ }^{2,3}$ The third set corresponds to a relatively wide microstrip. ${ }^{6}$ The typical values of film resistance and microwave frequencies used in CPW-FMR are shown as a gray rectangle. The phenomenon of shielding appear to play an important role in most CPW-FMR experiments: the electrical field is expected to be completely shielded in

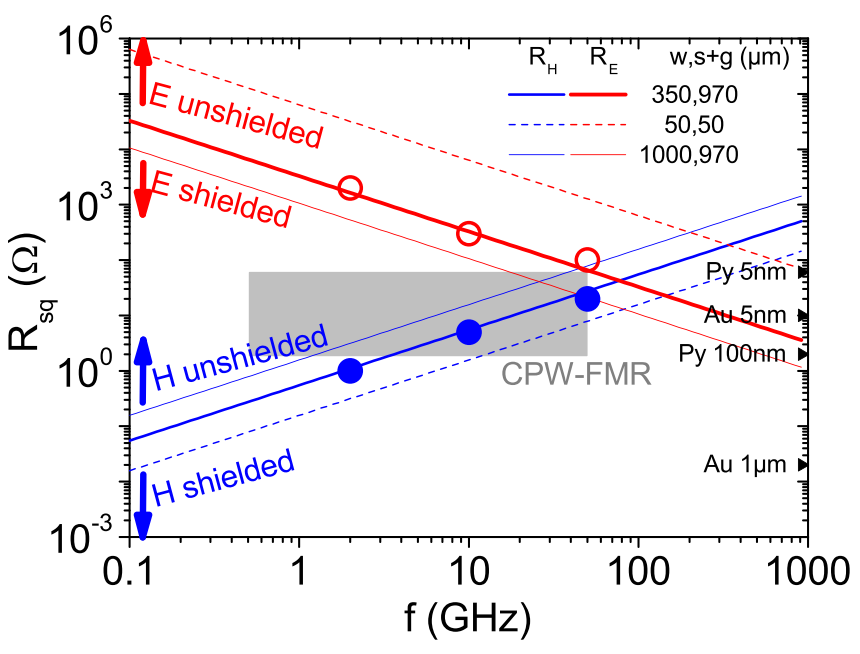

FIG. 3. Phase diagram for the phenomenon of microwave shielding. The threshold values $R_{E}$ (red line) and $R_{H}$ (blue line) are plotted as a function of the microwave frequency for three geometries of transmission lines. The dots show the values obtained from the simulation of Fig. 1 at 2, 10, and $50 \mathrm{GHz}$. Typical square resistance for ferromagnetic metal films (Py) and non-magnetic films $(\mathrm{Au})$ are shown on the right.

nearly all cases, while the magnetic field is expected to be shielded in the thick film, high frequency, wide-CPW sector of the parameter space. Once again, we emphasize that this phenomenon of microwave shielding occurs for film thickness much smaller than the relevant skin depth. It actually appears as soon as the film resistance is small enough to allow for shunting currents to flow in it. It is therefore expected not to depend strongly on the simplifying assumptions of the model of Fig. 1 (film substrate of dielectric constant unity, metal box, film explicitly connected to the ground). Once shielded, the electric and magnetic fields are indeed concentrated in the air gap separating the CPW and the film, so the properties of the film substrate are not of major importance. Moreover, even in the absence of an explicit ground connection of the film (films can be electrically floating in CPW-FMR experiments), the large capacitive coupling between the CPW ground planes and the film is expected to provide the required low impedance paths. Let us also comment on the dependence of the effect on the film height $h$. In Fig. 1(a), we have chosen a relatively high value of $30 \mu \mathrm{m}$ because this facilitates the finite element simulations and the visualization of the modes. In most CPW-FMR experiments the film is placed directly above the film and its height is probably only limited by the relative planarities of the film and CPW top surface. The distance between the top of the center track and the film is therefore not expected to exceed a few micrometers (enough to avoid a direct short circuit). The main difference is that $Z_{c}^{P P}$-which scales as $h / w$ - can now be very low.

Let us finally examine the consequences of the phenomenon of shielding in broadband FMR experiments. (i) In the magnetic shielding regime, the currents $j_{H}$ govern the distribution of the magnetic field across the film thickness. One gets a linear decrease to zero in the case of a single metal film of moderate conductance, ${ }^{9}$ or more complicated distributions in the case of multilayers containing metal films of different conductivities. ${ }^{16}$ Due to such strong inhomogeneity of the magnetic field, non-uniform resonance modes such as 
perpendicular standing spin wave modes or optical modes in magnetic bilayers can be excited, ${ }^{6,10}$ in contrast with the unshielded case where the only mode excited is the uniform FMR. Moreover, in the multilayer case, non-magnetic metal layers (capping and spacers in magnetoresistive stacks or heavy metals in spin-Hall stacks) can influence drastically the profile and amplitude of the magnetic field acting onto the ferromagnetic layers. ${ }^{16}$ (ii) Shielding also influences the amplitude and phase of the CPW-FMR signal through its influence on the microwave propagation characteristics $Z_{c}$ and $n_{\text {eff. }}$ To illustrate this, one can write the change of the $S_{i j}$ parameters $(i, j=1,2)$ of a section of length $l$ of the loaded CPW as $\delta S_{i j}=P_{i j} i \omega l \delta L / 2 Z_{0}$, where $\delta L=\eta \mu_{0} \chi t_{\text {film }} / w$ is the change of inductance per unit length of the loaded transmission line due to FMR. Here, $\chi$ is the FMR magnetic susceptibility of the film, $\eta$ is a dimensionless parameter related to the transverse distribution of the magnetic field, ${ }^{20}$ and $P_{i j}$ are coefficients which can be calculated using the $T$ matrix formalism. ${ }^{12}$ Due to multiple reflections at the loaded CPW/unloaded CPW interfaces, the $P_{i j}$ 's show strong resonances as a function of the propagation phase $2 \pi f n_{e f f} l / c$ as soon as the two characteristic impedances do not match, which is precisely what is expected to happen in the presence of shielding. For a reliable broadband extraction of $\delta L$, one has to eliminate these resonances. This can be done either by suitably post-processing the $S$ parameters (see, e.g., the deembedding procedure in Ref. 3, note that full 3D electromagnetic simulations could also be useful for modeling accurately the transition between loaded and unloaded parts of the transmission line), or by compensating this change of impedance in the design of the CPW, e.g., by tapering the central track to a much smaller width under the film, so that the shielded characteristic impedance becomes $50 \Omega$. With this last option, the CPW-FMR signal should become much more easily readable and also somehow stronger (because of the $1 / w$ dependence of $\delta L$ ).

To conclude, this paper provides an analysis of the propagation of electromagnetic waves along a coplanar waveguide over which a conducting film is placed. A full wave 2D electromagnetic simulation in a well controlled channelized coplanar waveguide geometry shows that such a film is able to shield completely the electric and/or the magnetic field of the CPW depending on the value of its square resistance. These results are interpreted quantitatively with the help of a simple distributed impedance model accounting for the shielding currents likely to flow along the metal film. This model allows one to predict the range of parameters for which shielding of the electric and/or magnetic fields is expected to occur. From this, it is concluded that the phenomenon should be accounted for in the analysis of most broadband inductive measurements of ferromagnetic resonance performed on metal ferromagnetic films. Although microwave shielding may appear as a detrimental complication for the users of CPW-FMR, we believe that it can be accounted quite easily for using the tools given in this letter. Data analysis routines and/or CPW designs compensating for the impedance mismatch could certainly be developed in the future. This will allow to improve the performances of the technique in terms of bandwidth, sensitivity and signal readability. A proper modeling of the magnetic shielding in multilayers could also be of major importance for understanding broadband FMR measurements on spintronics film stacks.

The author would like to thank O. Bengone and C. Tugene (IPCMS) for their assistance with the simulations, B. Rosas and D. Leavitt (Southwest Microwave) and C. Candun (Elexo) for providing test boards, J. Will (IUT Louis Pasteur) for preliminary measurements and Y. Henry, I. Camara (IPCMS), and O. Tantot (XLIM) for useful discussions. This work was supported by the ANR (NanoSWITI, ANR-11-BS10-003).

${ }^{1}$ Y. Ding, T. J. Klemmer, and T. M. Crawford, J. Appl. Phys. 96, 2969 (2004).

${ }^{2}$ J.-M. L. Beaujour, W. Chen, K. Krycka, C.-C. Kao, J. Z. Sun, and A. D. Kent, Eur. Phys. J. B 59, 475 (2007).

${ }^{3}$ C. Bilzer, T. Devolder, P. Crozat, C. Chappert, S. Cardoso, and P. P. Freitas, J. Appl. Phys. 101, 074505 (2007).

${ }^{4}$ S. S. Kalarickal, P. Krivosik, M. Wu, C. E. Patton, M. L. Schneider, P. Kabos, T. J. Silva, and J. P. Nibarger, J. Appl. Phys. 99, 093909 (2006).

${ }^{5}$ T. Devolder, P.-H. Ducrot, J.-P. Adam, I. Barisic, N. Vernier, J.-V. Kim, B. Ockert, and D. Ravelosona, Appl. Phys. Lett. 102, 022407 (2013).

${ }^{6}$ K. J. Kennewell, M. Kostylev, N. Ross, R. Magaraggia, R. L. Stamps, M. Ali, A. A. Stashkevich, D. Greig, and B. J. Hickey, J. Appl. Phys. 108, 073917 (2010).

${ }^{7}$ T. J. Silva, C. S. Lee, T. W. Crawford, and C. T. Rogers, J. Appl. Phys. 85, 7849 (1999).

${ }^{8}$ G. Counil, J.-V. Kim, T. Devolder, C. Chappert, K. Shigeto, and Y. Otani, J. Appl. Phys. 95, 5646 (2004).

${ }^{9}$ M. Kostylev, J. Appl. Phys. 106, 043903 (2009).

${ }^{10}$ M. Kostylev, A. A. Stashkevich, A. O. Adeyeye, C. Shakespeare, N. Kostylev, N. Ross, K. Kennewell, R. Magaraggia, Y. Roussigne, and R. L. Stamps, J. Appl. Phys. 108, 103914 (2010).

${ }^{11} \mathrm{~B}$. Rosas, "The design and test of broadband launches up to $50 \mathrm{GHz}$ on thin and thick substrates," Technical Report, Southwest Microwave, Inc., 2011, available at http://mpd.southwestmicrowave.com/resources/.

${ }^{12}$ S. Ramo, J. R. Whinnery, and T. Van Duzer, Fields and Waves in Communication Electronics, 2nd ed. (Wiley, New York, 1984).

${ }^{13}$ T. Cheung and J. Long, IEEE J. Solid-State Circuits 41, 1183 (2006).

${ }^{14}$ H. Hasegawa and H. Okizaki, Electron. Lett. 13, 663 (1977).

${ }^{15}$ S. Seki and H. Hasegawa, Electron. Lett. 17, 940 (1981).

${ }^{16}$ I. S. Maksymov and M. Kostylev, J. Appl. Phys. 113, 043927 (2013).

${ }^{17}$ In order to limit the number of mesh elements, the metal film is modeled as a relatively thick layer $\left(t_{\text {film }}=10 \mu \mathrm{m}\right)$ with a conductivity $\sigma=\left(R_{s q} t_{\text {film }}\right)^{-1}$. It was checked that the result of the simulation does not change when $\mathrm{t}_{\text {film }}$ is decreased to more realistic values while $\mathrm{R}_{\mathrm{sq}}$ is fixed.

${ }^{18} \mathrm{High}$ values of $E$ and $H$ are also observed in the substrate immediately below the center track. This is typical for a CCPW with a relatively thin substrate which represents a situation intermediate between a conventional CPW and a microstrip.

${ }^{19} \mathrm{We}$ use $Z=Z_{c} n_{e f f} \omega / c$ and $Y=n_{\text {eff }} \omega /\left(c Z_{c}\right)$ to relate the series impedance $Z$ and parallel admittance $Y$ per unit length to $Z_{c}$ and $n_{\text {eff }}$ for the CPW and PP modes and for their combination. ${ }^{12}$

${ }^{20}$ The parameter $\eta$, which can be seen as a filling factor, can be deduced from the 2D FEM simulations. For the design of Fig. 1, we find 0.2 in the magnetic shielding regime and 0.1 otherwise. 